\title{
Current and future treatments for parathyroid carcinoma
}

\author{
Kristin L Long ${ }^{1} \&$ Rebecca S Sippel ${ }^{*, 1}$ \\ ${ }^{1}$ Division of Endocrine Surgery, Department of Surgery, University of Wisconsin School of Medicine \& Public Health, Madison, WI \\ 53792, USA \\ *Author for correspondence: Tel.: +60 8263 1387; Fax: +60 8252 0912; sippel@surgery.wisc.edu
}

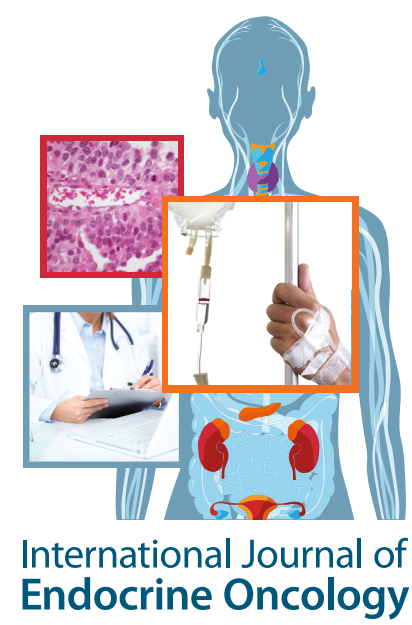

Parathyroid carcinoma (PC) is a rare disease and presents clinical challenges in diagnosis, management and adjuvant treatment. Rarely diagnosed preoperatively, PC is often discovered in the treatment of primary hyperparathyroidism. PC is predominantly treated surgically with minimal additional treatments available. Given the rarity of the disease, current and future efforts, as discussed in this article, are focused on increasing preoperative diagnosis, recognizing genetic patterns and pathologic markers and delineating nonsurgical options for patients with advanced disease.

First draft submitted: 13 April 2017; Accepted for publication: 17 January 2018; Published online: 7 February 2018

Parathyroid carcinoma (PC) is an extraordinarily rare endocrine malignancy found in approximately $1 \%$ of patients diagnosed with primary hyperparathyroidism [1]. Similar to benign presentation of primary hyperparathyroidism (PHPT), parathyroid carcinoma can present with symptoms of hypercalcemia, renal failure, nephrolithiasis, osteoporosis and cardiac or neurocognitive dysfunction [2]. Generally, preoperative-imaging modalities are unhelpful in distinguishing between benign and malignant parathyroid disease. Dramatic elevations in serum calcium or parathyroid hormone $(\mathrm{PTH})$ or presentation with hypercalcemic crisis may increase the suspicion of a diagnosis of PC, however, in most cases the diagnosis of PC is made intraoperatively. Given the rarity of PC, controversies exist regarding management, staging and follow-up [3]. Improved data regarding treatment outcomes and prognosis as well as the first consensus staging guidelines for PC have been recently published $[4,5]$. Due to the rarity of the disease, limited opportunities for large-scale studies exist, making advances in treatment quite difficult.

\section{Incidence}

The incidence of PC varies by country, from $1 \%$ of PHPT patients in the USA up to 5\% of PHPT patients in Japan [6]. Likewise, survival after diagnosis ranges from 1 month to 20 years or more [1,6]. In contrast to PHPT, which demonstrates a strong female predominance, $\mathrm{PC}$ appears to have no gender preference, and frequently occurs in younger patients than benign PHPT [6]. A total of 5- and 10-year survival rates for PC have been estimated between $78-85 \%$ and $49-70 \%$, respectively $[4,7-8]$. Disease recurrence is extremely common, occurring in over $50 \%$ of patients [9]. Metastases to lymph nodes are rare, and unfortunately, most patients who die of PC do so from complications of unrelenting hypercalcemia due to distant metastatic disease [10].

\section{Presentation \& diagnosis}

PC presents in a manner similar to PHPT with an elevated serum calcium level and an unsuppressed serum PTH level. PC is often considered when the serum calcium is markedly elevated (>14 mg/dl), but this is certainly not present in all cases. The patients demonstrate marked variety in their laboratory abnormalities and in their symptom profiles. Symptoms attributed to PHPT include, but are not limited to, bone and joint pain, muscle fatigue, difficulty sleeping, abdominal pain, reflux, depression and anxiety. Long-term effects of hypercalcemia can contribute to cardiovascular disease, including hypertension, and many patients show significant symptom improvement after surgical treatment of their parathyroid disease.

PC is rarely diagnosed preoperatively. Suspicion for PC arises when patients present with severe hypercalcemia $(>14 \mathrm{mg} / \mathrm{dl}$ or $>3.5 \mathrm{mmol} / \mathrm{l}$ ), marked PTH elevations, or demonstrate a palpable neck mass [2,6]. Most patients with PC have single-gland disease and multigland disease is uncommon. Occasionally imaging findings may suggest 


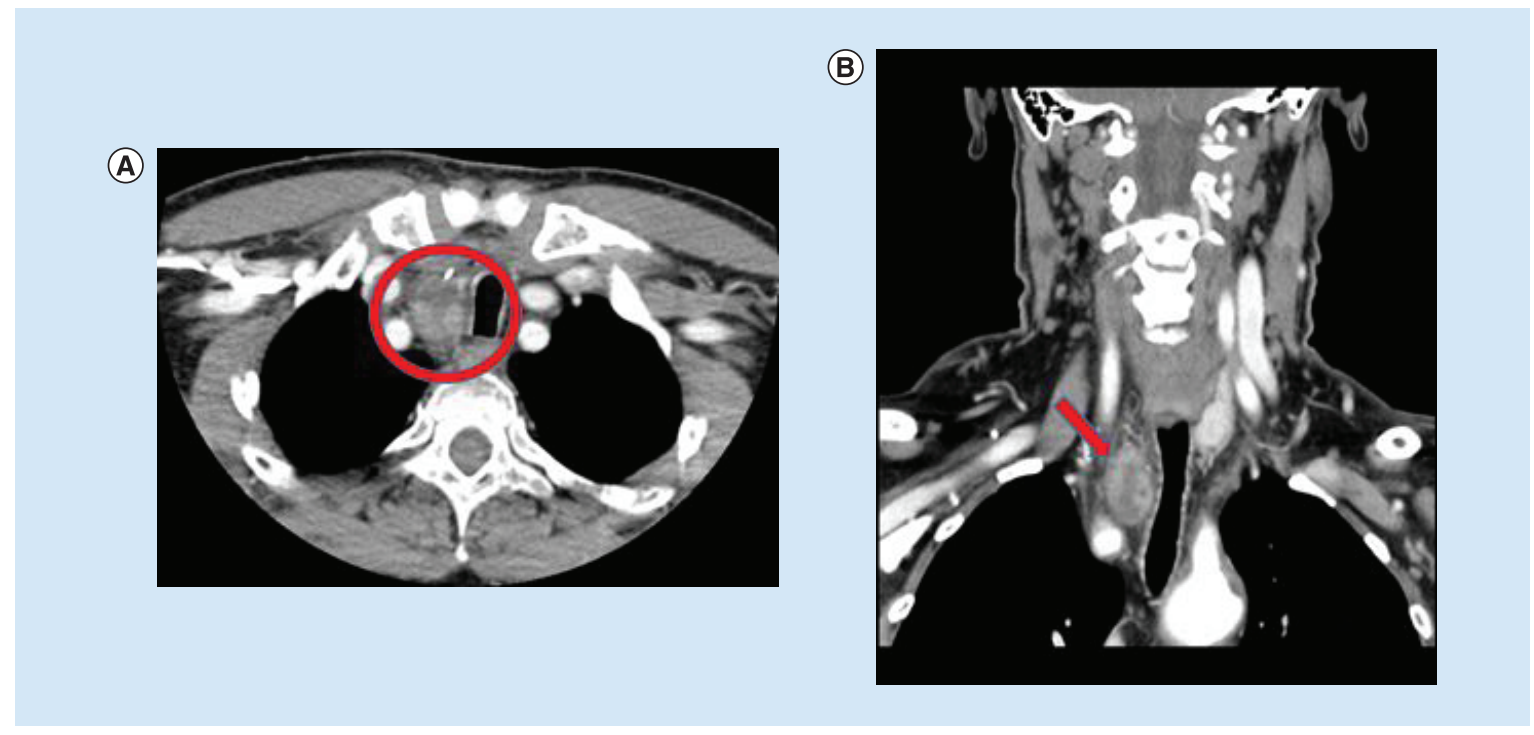

Figure 1. Grossly enlarged parathyroid gland with concern for local soft tissue invasion. (A) Transverse view demonstrating parathyroid gland with possible tracheal invasion; (B) Coronal view also suggesting possible tracheal involvement by parathyroid mass.

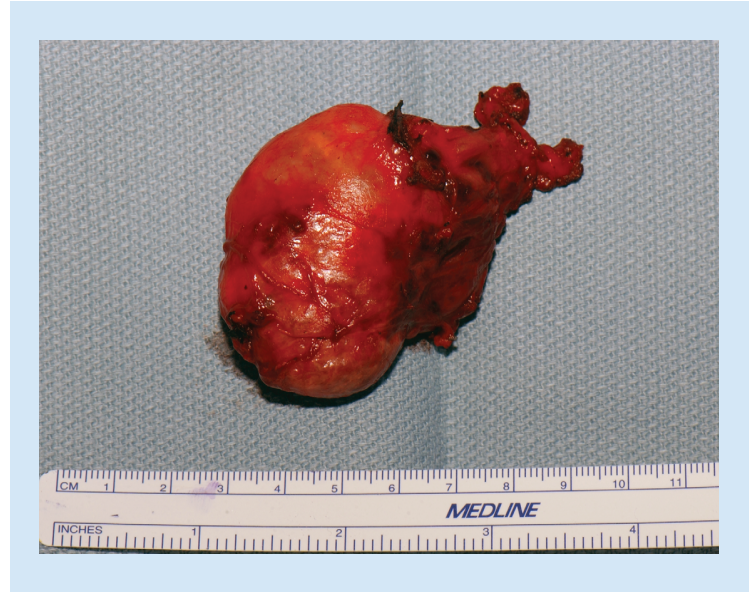

Figure 2. Parathyoid carcinoma attached to the adjacent thyroid lobe.

tumor invasion and raise a suspicion of malignancy as well (Figure 1). Invasion of surrounding structures at the time of operation also points toward a diagnosis of PC [4]. PC tumors are frequently described as firm, fixed and gray-white in color on gross appearance (Figure 2). Unfortunately, this is not universally true, as the affected gland may appear indistinguishable from a benign parathyroid adenoma [11]. In some cases, patients will present with classic symptoms of PHPT, ranging from nephrolithiasis to osteoporosis to mild systemic complaints and preoperative examination may yield no clues of an underlying malignancy. PC can occasionally be nonfunctional, as well. The patients may present at slightly younger ages than with traditional PHPT, but in general, few features distinguish benign disease from malignancy.

\section{Imaging}

Multiple radiologic techniques exist for preoperative localization in parathyroid disease, including ultrasound, Tc-99m sestamibi scans and high-resolution computed tomography. Preoperative imaging is not necessary to make a diagnosis of PHPT, and should only be used to guide surgical planning after the diagnosis has been established. Practice patterns vary, but many high-volume endocrine surgeons perform surgeon-directed ultrasound with excellent accuracy in identifying enlarged glands [12,13]. When ultrasound localization fails to identify an abnormal parathyroid gland, additional imaging such as Tc-99m-sestamibi or high-resolution 4D-computed tomography of 
the neck may be performed to further aid in localization. Cross-sectional imaging may be particularly helpful in identifying ectopic parathyroid glands, or those in locations poorly accessible to ultrasound (posterior glands deep in tracheoesophageal groove). Despite the modality used, $\mathrm{PC}$ is rarely differentiated from benign parathyroid disease on preoperative imaging. Ultrasound probably has the greatest yield in raising a suspicion for PC. Ultrasound may show a heterogeneous cystic structure, irregular borders or signs of local invasion [1]. Fine-needle aspiration of a potential PC should be avoided, as there is a documented risk of tumor seeding along the needle track [14,15]. If clinical evaluation and imaging leads to a high suspicion of PC, additional preoperative imaging with 18F-FDG PET/CT may help to clarify the extent of disease and gauge the extent of operation that would be needed [16]. If imaging suggests possible PC, then the need for a potential larger resection should be discussed with the patient prior to surgery.

\section{Genetics}

The first hereditary syndrome linked to PC was hyperparathyroidism-jaw tumor syndrome, derived from inactivating mutations in the gene CDC73 (previously known as HRPT2) [17]. These mutations can occur in over $75 \%$ of confirmed (by recurrence or metastases) PC, and more importantly, only very rarely (less than $1 \%$ of the time) are found in benign parathyroid disease [18]. It has been recommended that all patients with PC should be considered for germline testing for HRPT2. The HRPT2 gene is a tumor suppressor that codes for a protein called parafibromin. Since parafibromin expression can be detected via immunohistochemistry (IHC), it can be used to test for parafibromin expression and identify patients with HRPT2 mutations [18]. HRPT2 mutations have been linked not only to the presence of parathyroid carcinoma but also to the risk of disease recurrence [19].

As PC is a very rare disease, genetic and epigenetic studies may hold the most promise for future developments. Gene alterations between benign parathyroid adenomas and parathyroid carcinomas have been identified [20]. Large-scale epigenetic studies have demonstrated repression of miRNAs in PC tissue relative to normal parathyroid glands as well, arguing that further studies in epigenetic deregulation of parathyroid tumors may provide new strategies for treatment [21].

\section{Pathology}

Hyperparathyroidism can be caused by a variety of parathyroid neoplasms, and distinguishing PC from other tumors, such as adenomas, hyperplasia or atypical adenomas can be challenging. PC exists on a spectrum of parathyroid disease, ranging from parathyromatosis to atypical parathyroid neoplasms, to carcinoma. Parathyromatosis, defined as miliary seeding of benign parathyroid tissue into surrounding muscle and soft tissue, can occur from embryonic rests of parathyroid tissue or, more frequently, from iatrogenic spillage of parathyroid tissue [22]. This may appear as small, white plaques of disease, or may not be visible until final pathologic analysis of a specimen is completed. Parathyromatosis typically presents as recurrent disease with mild calcium elevations in patients who have had prior parathyroid surgery (particularly subtotal resections for secondary hyperparathyroidism) and can be confused with PC [23]. Atypical parathyroid adenomas may have features similar to PC but do not demonstrate invasive characteristics. In order to clearly diagnose PC on an initial specimen, evidence of lymphovascular or local invasion should be present [1]. PC can also be diagnosed clinically, when there is evidence of disease recurrence or the presence of distant metastatic disease.

PC may demonstrate a uniform appearance in a nodular or trabecular pattern. It is frequently characterized by the presence of dense fibrotic bands. Mitosis, and specifically atypical mitotic figures are frequently present. Diffuse nuclear enlargement and macronucleoli and an elevated Ki-67 also suggest malignancy (Figure 3).

Immunohistochemistry staining can assist in the differentiation of PC from benign parathyroid disease. The most commonly used marker for PC is parafibromin, which is encoded by the HRPT2 gene. When this gene is mutated, a loss of parafibromin expression and loss of staining is seen, making it a highly specific test for PC [24]. Parafibromin IHC demonstrates a decrease in specificity when atypical parathyroid neoplasms are included in the study samples, leading some authors to recommend standardized IHC protocols and scoring systems for parathyroid tissue [25]. Loss of APC or elevation of MIB-1 proliferation indices (the target marker for identifying Ki67) are additional markers that may aid in the pathologic diagnosis of PC [24]. In general, Ki67 of greater than $5 \%$ suggests PC, and overall, a panel of IHC analysis is considered more accurate than any single marker in diagnosing PC. In addition to diagnosis, molecular techniques can be helpful in assessing prognosis for PC patients. Downregulation of calcium-sensing receptor expression and a global loss of parafibromin staining have been shown to be negative determinants for prognosis of patients with parathyroid carcinoma [26]. 

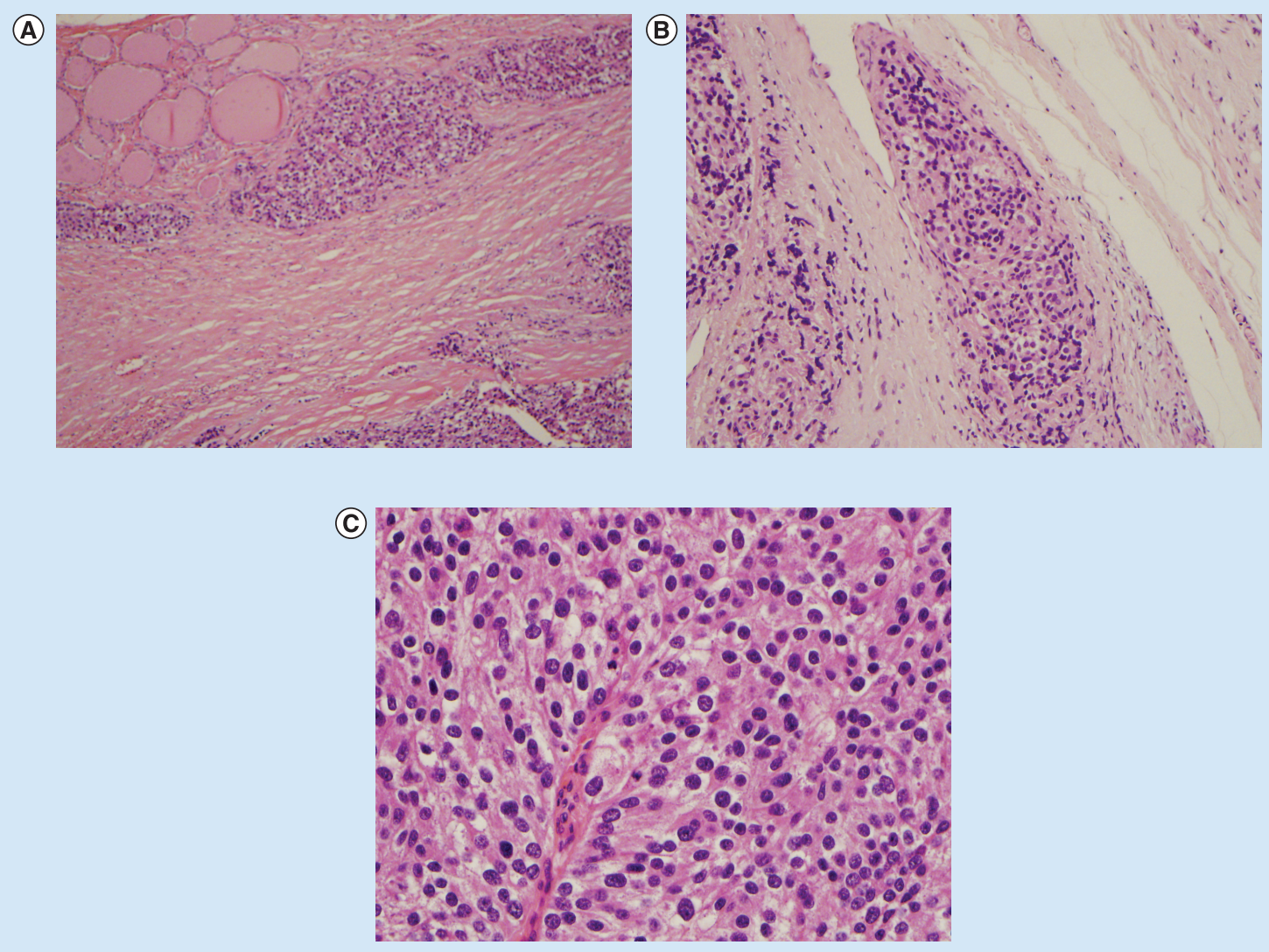

Figure 3. Microscopic characteristics of parathyroid carcinoma. (A) Parathyroid carcinoma (magnified $\times 100$ ) with a background of thick fibrous bands. Nests of parathyroid carcinoma cells are infiltrating the adjacent thyroid. (B) Parathyroid carcinoma showing vascular invasion in the center of the field (magnified $\times 200$ ). (C) Parathyroid carcinoma showing two mitotic figures in the center of the field (magnified $\times 400$ ). Reproduced with permission from Ricardo Lloyd.

\section{Surgery}

Surgical treatment is the mainstay for PC, and adequate initial operations should involve en bloc resection of the tumor with any involved structures [1-2,4,7-9,27-28]. Recognition of possible malignancy is imperative, as many cases of PC will be diagnosed intraoperatively. Even more likely is an unexpected postoperative diagnosis of PC, explaining why initial en bloc resection may be performed in as little as $12 \%$ of cases [7-8,29]. In order to optimize treatment of PC, it is essential for the surgeon to have a high index of suspicion for cancer when the inoperative findings are not what they would expect for benign disease. Careful attention to the tumor and preventing rupture of the specimen is paramount to minimize poor long-term outcomes [30]. Resection frequently involves the affected parathyroid gland, the ipsilateral thyroid lobe and involved muscle tissue. Occasionally, more invasive tumors may necessitate tracheal resections, or may involve portions of the esophagus, blood vessels or bone. If there is a suspicion of PC, frozen section is often unable to clarify the diagnosis. If the surgeon is suspicious for PC, it is best to just proceed with an oncologic en bloc resection. The key is to ensure that the capsule is maintained and that a complete resection is performed. As with surgery for benign parathyroid disease, use of intraoperative PTH measurements have been effective in demonstrating removal of all hyperfunctioning parathyroid tissue [31].

Significant controversy exists over central neck dissection (CND) for PC. While en bloc resection results in fewer reoperations for disease, limited data exist on the implications of prophylactic lymph node dissections for PC [32]. Lymph node disease is infrequent, and recurrences in other soft-tissue structures of the neck are more common [33,34]. Retrospective data have demonstrated no difference in rates of metastases and death between patients who did or did not have lymph nodes examined [10]. Tumors larger than $3 \mathrm{~cm}$ had a greater incidence of lymph node metastases, and larger studies may help to identify select populations that would benefit from 
prophylactic CND in addition to en bloc tumor removal [10]. Currently, CND in patients with PC and clinically negative lymph nodes offers minimal therapeutic benefits, and must be weighed carefully against the known risks of such procedures. The benefits of doing a prophylactic CND, in some patients, is likely related to the removal of the surrounding fibrofatty tissue and not really related to the removal of nodal tissue.

\section{Staging: new AJCC}

In early 2017, the newest edition of the American Joint Committee on Cancer guidelines introduced a staging system for PC [5]. Acknowledging that adequate identification of significant prognostic factors has been challenging due to mostly retrospective and single-institution studies, the panel showed a demonstrable difference in cancerspecific survival between patients with disease localized to the neck and those with distant metastatic disease. The proposed registry data collection variables for PC included age at diagnosis, gender, race, size of primary tumor, location of tumor, presence of invasion into the surrounding structures, distant metastatic disease, number of lymph nodes removed, number of positive lymph nodes, highest preoperative calcium, highest preoperative PTH, presence of lymphovascular invasion, histologic grade (high grade or low grade), weight of primary tumor, mitotic rate and time to recurrence. The TNM definitions for PC include atypical parathyroid neoplasm (Tis), a common pathologic result that may confound treatment strategies. T1 tumors are localized to the parathyroid gland with extension limited to the soft tissue, while T2-T4 tumors directly invade the thyroid gland (T2), surrounding structures such as recurrent laryngeal nerve, esophagus, or trachea (T3) or major blood vessels/spine (T4). Lymph node disease is quantified by the absence (N0) or presence of disease in the central (N1a) or lateral (N1b) neck lymph nodes. Likewise, distant metastatic disease is described by the absence of disease outside of the neck (M0) versus evidence of disease not confined to the neck (M1).

\section{Adjuvant treatment}

Adjuvant treatment of PC is extremely limited. In the past, small cohorts of patients have undergone external beam radiation therapy for treatment of disease considered unresectable, however utility of this is questioned as a fair number of PC tumors are considered radioresistant [35-37]. No benefit for overall survival or local recurrence has been clearly identified, and use of radiotherapy is often restricted to palliative treatment of advanced disease or in the presence of distant disease [4,7,36-37]. Cytotoxic chemotherapy is used even less frequently than external beam radiation therapy, and only isolated reports with very small numbers of patients exist [37,38]. Reports of successful radiofrequency embolization or transarterial catheter embolization of distant metastatic disease exist, but the number of cases in which it has been used is limited [39,40].

The greatest challenge in the management of metastatic disease is management of the associated hypercalcemia. Management of refractory hypercalcemia due to metastatic PC has included bisphosphonates and calcimimetic agents, however denosumab, a human monoclonal antibody against RANKL which inhibits osteoclast survival, has also been suggested as a safe alternative [41-44]. Unfortunately, as with most medical therapies, treatment effect weakens over time and each of these options are unlikely to provide long-term relief. Limited efficacy of immunotherapy is reported $[45,46]$. In general, many adjuvant therapies are used anecdotally with no consensus on efficacy or response. Overall goals remain to minimize effects of hypercalcemia and control disease burden, and each case of advanced PC must be personalized.

First-line treatment of hypercalcemia is similar to any hypercalcemic crisis and includes hydration and bisphosphonates (typically zoledronic acid or pamidronate). For patients that do not respond to hydration and bisphosphonates, calcimimetics can be used. Cinacalcet, a long-acting calcimimetic drug, is approved by the US FDA for the treatment of hypercalcemia in PC. The initial dose of cinacalcet is $30 \mathrm{mg}$ twice-daily and the dose can be increased every 2-4 weeks, based on drug tolerance and serum calcium levels. Nausea is the most common dose-limiting side effect.

\section{Surveillance/follow-up}

After an initial surgical resection, the patients should be followed carefully with both regular laboratory testing for both calcium and PTH levels as well as neck ultrasounds. Long-term survival is possible, and routine surveillance can help identify early recurrence of disease [46,47]. Disease recurrence can be treated with surgical resection, if it is anatomically feasible. Many patients with PC may undergo multiple resections, both for disease in the neck as well as for distant metastatic disease. Hepatic and pulmonary metastases have been successfully resected as palliative measures $[47,48]$. Complete clearance of disease remains the ultimate goal, and surgery remains the most effective 
treatment for recurrent disease. Given the hypercalcemia associated with recurrent/metastatic disease, there is a role for even noncurative surgical debulking as a mechanism to help facilitate the management of hypercalcemia.

For patients with known germline mutations of HRPT2, routine surveillance with annual calcium, PTH and $25-\mathrm{OH}$ vitamin $\mathrm{D}$ levels as well as periodic neck ultrasound (to assess for the rare but reported detection of nonfunctioning PC) are suggested. Given the development of jaw tumors, a dental panorex image every 5 years is also recommended [49]. In patients with diagnosed PC, lifelong surveillance is recommended [50,51].

Follow-up recommendations for patients with atypical parathyroid adenomas remains less clear, as the behavior of this heterogeneous group of tumors is less well defined. In general, atypical parathyroid adenomas are often treated more like patients with a malignant diagnosis, in order to eliminate missing aggressive or recurrent disease. These patients should be followed closely to identify signs of recurrence. Since most tumors produce PTH and lead to hypercalcemia, routine lab testing is a simple way to follow these patients on a routine basis.

\section{New developments/future treatment}

In addition to medical treatment with calcimimetics and denosumab, experimental immunotherapy has reportedly caused tumor shrinkage in isolated cases of advanced PC [45,46]. Importantly, this treatment modality offers potential tumor shrinkage in addition to control of the refractory hypercalcemia. In patients with metastatic or advanced $\mathrm{PC}$, death is most frequently from effects of severe hypercalcemia rather than direct tumor invasion. True efficacy of this treatment remains unknown, however, as only anecdotal case reports exist. Likewise, isolated reports of ethanol ablation temporarily decreasing serum calcium and PTH levels suggest use of ultrasound-guided injection for palliation of PC in selected cases [50]. This modality, however, should be reserved for disease outside of the central neck, as ethanol injection in the central neck can permanently injure a functioning recurrent laryngeal nerve.

Advanced techniques such as whole-exome sequencing have been employed in an attempt to identify genetic abnormalities in PC [51]. These studies have reinforced the importance of $C D C 73$, but additionally, recurrent germline and somatic mutations in prune homolog 2 (PRUNE2) were found in a significant percentage of PC samples [51]. Likewise, amplification of CCND1, a gene that encodes cyclin D1, may be implicated in the molecular pathogenesis of PC as it was found to be significantly more prevalent in cases of PC than benign parathyroid adenomas [52]. Complete genomic analysis of both primary and recurrent tumor samples is available for one patient, and this demonstrated a loss of PIK3CA activation during tumor evolution, and offered insight into several potential new diagnostic or therapeutic targets first identified as somatic mutations [53]. In order to practically apply this information, large-scale studies with PC patients will be necessary. As a rare disease, advances in treatment for PC are difficult to develop and study.

Finally, improving the ability of surgeons to recognize PC intraoperatively, and therefore to perform the appropriate initial operation, is vital to increasing survival for PC patients. Awareness of the disease and its possible features as well as significant experience in parathyroid surgery is the best option to minimize substandard care and maximize successful outcomes for patients.

\section{Conclusion \& future perspective}

PC remains a rare entity, making widely applicable studies about prognosis, treatment or recurrence difficult. Adequate surgical resection is the best initial treatment, with en bloc resection of all tumor and involved structures necessary for optimal outcomes. Preoperative diagnosis remains challenging, mandating surgeons maintain a high index of suspicion for possible malignancy during parathyroid operations. Adjuvant treatment options are sporadically used at best, and further study and innovation is needed to improve options and outcomes. In the coming years, early recognition, appropriate initial surgical treatment and close surveillance should be emphasized as further research works to identify unique adjuvant therapies for PC. Given the rarity of the disease, collaborative research will be vital to advance treatment options.

Financial \& competing interests disclosure

The authors have no relevant affiliations or financial involvement with any organization or entity with a financial interest in or financial conflict with the subject matter or materials discussed in the manuscript. This includes employment, consultancies, honoraria, stock ownership or options, expert testimony, grants or patents received or pending, or royalties.

No writing assistance was utilized in the production of this manuscript. 


\section{Executive summary}

- Parathyroid carcinoma (PC) should be suspected with markedly elevated serum calcium levels (>14 mg/dl or $>3.5 \mathrm{mmol} / \mathrm{l})$.

- Fine needle aspiration should be avoided if there is a suspicion of PC.

- Surgical treatment should involve en bloc resection of all involved tissue, and is best performed by high-volume parathyroid surgeons.

- Few adjuvant therapies currently exist for treatment of recurrent or metastatic PC.

- Patients with PC should have lifelong surveillance.

- Management of refractory PC often centers around optimizing treatment of hypercalcemia.

\section{Open access}

This work is licensed under the Creative Commons Attribution 4.0 License. To view a copy of this license, visit http://creativecomm ons.org/licenses/by/4.0/

\section{References}

1. Harari A. Parathyroid carcinoma. In: Textbook of Endocrine Surgery (3rd Edition). Clark OH, Duh Q-Y, Kebebew E, Gosnell JE, Shen WT (Eds). Jaypee Brothers Medical Publishers, New Delhi, India, 927-935 (2016).

2. Cetani F, Pardi E, Marcocci C. Update on parathyroid carcinoma. J. Endocrinol. Invest. 39, 595-606 (2016).

3. Medas F, Erdas E, Loi G et al. Controversies in the management of parathyroid carcinoma: a case series and review of the literature. Int. J. Surg. 28, S94-S98 (2016).

4. Asare EA, Sturgeon C, Winchester DJ et al. Parathyroid carcinoma: an update on treatment outcomes and prognostic factors from the National Cancer Data Base (NCDB). Ann. Surg. Oncol. 22, 3990-3995 (2015).

5. Landry CS, Wang TS, Asare EA et al. AJCC cancer staging manual (8th Edition). Amin MB et al. (Eds). Chapter 75, 903-910 (2017).

6. Harari A, Waring A, Fernandez-Ranvier G et al. Parathyroid carcinoma: a 43-year outcome and survival analysis. J. Clin. Endocrinol. Metab. 96(12), 3679-3686 (2011).

7. Lee PK, Jarosek SL, Virnig BA, Evasovich M, Tuttle TM. Trends in the incidence and treatment of parathyroid carcinoma in the United States. Cancer 109, 1736-1741 (2007).

8. Hundahl SA, Fleming ID, Fremgen AM, Menck HR. Two hundred eight-six cases of parathyroid carcinoma treated in the US between 1985-1995: a National Cancer Data Base Report. Cancer 86, 538-544 (1999).

9. Sadler C, Gow KW, Beierle EA et al. Parathyroid carcinoma in more than 1,000 patients: a population-level analysis. Surgery 156(6), 1622-1630 (2014).

10. Hsu KT, Sippel RS, Chen H, Schneider DF. Is central lymph node dissection necessary for parathyroid carcinoma? Surgery 156(6), 1336-1341 (2014).

11. Sharretts JM, Kebebew E, Simonds WF. Parathyroid cancer. Semin. Oncol. 37(6), 580-590 (2010).

12. Javiev AA, Lew JI, Solorzano CC. Surgeon-performed ultrasound: a single institution experience in parathyroid localization. Surgery 146(4), 569-575 (2009).

13. Arora S, Balash PR, Yoo J, Smith GS, Prinz RA. Benefits of surgeon-performed ultrasound for primary hyperparathyroidism. Langenbecks Arch. Surg. 394(5), 861-867 (2009).

14. Wei CH, Harari A. Parathyroid carcinoma: update and guidelines for management. Curr. Treat. Options Oncol. 13(1), 11-23 (2012).

15. Agarwal G, Dhingra S, Mishra SK, Krishnani N. Implantation of parathyroid carcinoma along fine needle aspiration track. Langenbecks Arch. Surg. 391(6), 623-626 (2006).

16. Evangelista L, Sorgato N, Torresan F et al. FDG-PET/CT and parathyroid carcinoma: review of the literature and illustrative case series. World J. Clin. Oncol. 2(10), 348-354 (2011).

17. Carpten JD, Robbins CM, Villablanca A et al. HRPT2, encoding parafibromin, is mutated in hyperparathyroidism-jaw tumor syndrome. Nat. Genet. 32(4), 676-680 (2002).

18. Gill AJ. Understanding the genetic basis of parathyroid carcinoma. Endocr. Pathol. 25, 30-34 (2014).

19. Enomoto K, Uchino S, Ito A et al. The surgical strategy and the molecular analysis of patients with parathyroid cancer. World J. Surg. 34, 2604-2610 (2010).

20. Starker LF, Svedlund J, Udelsman R et al. The DNA methylome of benign and malignant parathyroid tumors. Genes Chromosomes Cancer 50(9), 735-745 (2011).

21. Verdelli C, Forno I, Vaira V, Corbetta S. Epigenetic alterations in human parathyroid tumors. Endocrine 49(2), 324-332 (2015). 
22. Melck AL, Carty SE, Seethala RR et al. Recurrent hyperparathryoidisim and forearm parathyromatosis after total parathyroidectomy. Surgery 148(4), 867-875 (2010).

23. Fernandez-Ranvier GG, Khanafshar E, Jensen K et al. Parathyroid carcinoma, atypical parathyroid adenoma, or parathyromatosis? Cancer 110(2), 255-264 (2007).

24. Hosny Mohammed K, Siddiqui MT, Willis BC et al. Parafibromin, APC and MIB-1 are useful markers for distinguishing parathyroid carcinomas from adenomas. Appl. Immunohistochem. Mol. Morphol. 25(10), 731-735 (2016).

25. Hu Y, Liao Q, Cao S, Gao X, Zhao Y. Diagnostic performance of parafibromin immunhistochemical staining for sporadic parathyroid carcinoma: a meta-analysis. Endocrine 54(3), 612-619 (2016).

26. Witteveen JE, Hamdy NA, Dekkers OM et al. Downregulation of CASR expression and global loss of parafibromin staining are strong negative determinants of prognosis in parathyroid carcinoma. Mod. Pathol. 24, 688-697 (2011).

27. Iihara M, Okamoto T, Suzuki R et al. Functional parathyroid carcinoma: long-term treatment outcome and risk factor analysis. Surgery 142(6), 936-943 (2007).

28. Lee YS, Hong SW, Jeong JJ et al. Parathyroid carcinoma: a 16-year experience in a single institution. Endocr. J. 57(6), $493-497$ (2010).

29. Young S, Wu JX, Li N, Yeh MW. More extensive surgery may not improve survival over parathyroidectomy alone in parathyroid carcinoma. Ann. Surg. Onc. 23, 2898-2904 (2016).

30. Villar Del Moral J, Jimenez Garcia A, Salvador Egea P et al. Prognostic factors and staging systems in parathyroid cancer: a multi-center cohort study. Surgery 156(5), 1132-1144 (2014).

31. Adam MA, Untch BR, Olson JA. Parathyroid carcinoma: current understanding and new insights into gene expression and intraoperative parathyroid hormone kinetics. Oncologist 15(1), 61-72 (2010).

32. Fernandez-Ranvier GG, Chau AH. Molecular tests for parathyroid carcinoma. In: Textbook of Endocrine Surgery (3rd Edition). Clark OH, Duh Q-Y, Kebebew E, Gosnell JE, Shen WT (Eds). Jaypee Brothers Medical Publishers, New Delhi, India, 939-953 (2016).

33. Talat N, Schulte KM. Clinical presentation, staging, and long-term evolution of parathyroid cancer. Ann. Surg. Oncol. 17(8), 2156-2174 (2010).

34. Kebebew E, Arici C, Duh QY, Clark OH. Localization and reoperation results for persistent and recurrent parathyroid carcinoma. Arch. Surg. 136(8), 878-885 (2001).

35. Goswamy J, Lei M, Simo R. Parathyroid carcinoma. Curr. Opin. Otolarygnol. Head Neck Surg. 24(2), 155-162 (2016).

36. Christakis I, Silva AM, Kwatampora LJ et al. Oncologic progress for the treatment of parathyroid carcinoma is needed. J. Surg. Onc. 144, 708-713 (2016).

37. Busaidy NL, Jimenez C, Habra MA et al. Parathyroid carcinoma: a 22-year experience. Head Neck 26(8), 716-726 (2004).

38. Witteveen JE, Haak HR, Kievit J, Morreau H, Romijn JA, Hamdy NA. Challenges and pitfalls in the management of parathyroid carcinoma: 17-year follow-up of a case and review of the literature. Horm. Cancer 1(4), 205-214 (2010).

39. Clayman GL, Gonzalez HE, El-Naggar A, Vassilopoulou-Sellin R. Parathyroid carcinoma: evaluation and interdisciplinary management. Cancer 100(5), 900-905 (2004).

40. Artinyan A, Guzman E, Maghami E et al. Metastatic parathyroid carcinoma to the liver treated with radiofrequency ablation and transcatheter arterial embolization. J. Clin. Oncol. 26, 4039-4041 (2008).

41. Fountas A, Andrikoula M, Giotaki $Z$ et al. The emerging role of denosumab in the long-term management of parathyroid carcinoma-related refractory hypercalcemia. Endocr. Pract. 21(5), 468-473 (2015).

42. Karuppiah D, Thanabalasingham G, Shine B et al. Refractory hypercalcemia secondary to parathyroid carcinoma: response to high-dose denosumab. Eur. J. Endocrinol. 171(1), K1-K5 (2014).

43. Tong CV, Hussein Z, Noor NM, Mohamad M, Ng WF. Use of denosumab in parathyroid carcinoma with refractory hypercalcemia. QJM 108, 49-50 (2015).

44. Owen RP, Silver CE, Pellitteri PK et al. Parathyroid carcinoma: a review. Head Neck 33(3), 429-436 (2011).

45. Betea D, Bradwell AR, Harvey TC et al. Hormonal and biochemical normalization and tumor shrinkage induced by anti-parathyroid hormone immunotherapy in a patient with metastatic parathyroid carcinoma. J. Clin. Endocrinol. Metab. 89(7), 3413-3420 (2004).

46. Horie I, Ando T, Inokuchi $\mathrm{N}$ et al. First Japanese patient treated with parathyroid hormone peptide immunication for refractory hypercalcemia caused by metastatic parathyroid carcinoma. Endocr. J. 57(4), 287-292 (2010).

47. Mezhir JJ, Melis M, Headley RC, Pai RK, Posner MC, Kaplan EL. Successful palliation of hypercalcemia secondary to metastatic parathyroid cancer: an unusual indication for hepatic resection. J. Hepatobiliary Pancreat. Surg. 14(4), 410-413 (2007).

48. van Haaren ER, Kievit J, Huysmans HA, van de Velde CJ. Successful resection of hepatic and pulmonary metastases in a patient with parathyroid carcinoma. Jpn. J. Clin. Oncol. 26(2), 99-102 (1996).

49. Sandelin K, Auer G, Bondeson L, Grimelius L, Farnebo LO. Prognostic factors in parathyroid cancer: a review of 95 cases. World J. Surg. 16(4), 724-731 (1992). 
50. Montenegro FL, Chammas MC, Juliano AG, Cernea CR, Cordeiro AC. Ethanol injection under ultrasound guidance to palliate unresectable parathyroid carcinoma. Arg. Bras. Endocrinol. Metabol. 52(4), 707-711 (2008).

51. Yu W, McPherson JR, Stevenson M et al. Whole-exome sequencing studies of parathyroid carcinomas reveal novel PRUNE-2 mutations distinctive mutational spectra related to APOBEC-catalyzed DNA mutagenesis and mutational enrichment in kinases associated with cell migration and invasion. J. Clin. Endocrinol. Metab. 100(2), E360-E364 (2015).

52. Zhao L, Sun LH, Liu DM et al. Copy number variation in CCND1 gene is implicated in the pathogenesis of sporadic parathyroid carcinoma. World J. Surg. 38(7), 1730-1737 (2014).

53. Kasaian K, Wiseman SM, Thiessen $\mathrm{N}$ et al. Complete genomic landscape of a recurring sporadic parathyroid carcinoma. J. Pathol. 230, 249-260 (2013). 
